\title{
Pharmacogenetic Perspective for Optimal Gout Management
}

\author{
Khalifa Alrajeh, PharmD, MSCR ${ }^{1}$, Youssef M. Roman, PharmD, Ph.D. ${ }^{1 *}$ \\ ${ }^{1}$ Department of Pharmacotherapy and Outcome Science, Virginia Commonwealth University, School of \\ Pharmacy, Richmond, Virginia, United States of America \\ 410 N $12^{\text {th }}$ Street \\ Richmond, Virginia 23298 \\ * Corresponding Author: romany2@vcu.edu
}

\begin{abstract}
Pharmacogenomics (PGx) is the study of how variations of the patient genetic makeup could influence the selection and dosing of medications, including urate-lowering therapies (ULTs). Patients with gout present with several comorbidities, warranting the use of several long-term medications that increase their pill burden and the risk of adverse drug events. PGx testing can identify individuals who are more or less likely to benefit from a given treatment and ultimately improve adherence and reduce polypharmacy. Gout management involves using various ULTs associated with specific genetic variations that could modulate medication safety and efficacy. Individuals carrying the $H L A-B * 58: 01$ variant are at a higher risk of serious and life-threatening skin reactions from taking allopurinol. Besides, racial disparities in HLA-B distribution warrant testing for its status in high-risk populations, specifically some Asian subgroups and African Americans. G6PD-deficient individuals are prone to develop hemolytic anemia and methemoglobinemia with pegloticase and probenecid use. Patients with the less active form of the drug-metabolizing CYP2C9 are at higher risk for NSAID-related upper gastrointestinal (GI) bleeding. The drug-gene pair interactions among various gout therapies were also reviewed. Allopurinol-related genes included $A O X, A B C G 2$, and $S L C 22 A 12$. Febuxostat-related genes included UGT1A1. Benzbromarone- and probenecid- related genes included $S L C 22 A 12$ and $A B C B 1$. Colchicine-related genes included CYP2D6, $A B C B 1$, rs6916345 G>A, and SEPHS1. Finally, the corticosteroid-related gene included HCG22; and the anakinra-related gene included IL1RA. This review synthesizes the most contemporary level of evidence for using PGx in gout pharmacotherapy, as referenced in the CPIC guidelines, FDA, Phar$\mathrm{mGKB}$, and current literature.
\end{abstract}

Keywords: Gout; Pharmacogenetics; Genetics; HLA-B*58:01; CYP2C9; Allopurinol; Urate-Lowering Therapy; NSAIDs; G6PD; Urate Transportome

\section{Background}

Pharmacogenomics (PGx) leverages patient genetics to ascertain the response to pharmacotherapy, including gout treatments. The inclusion of pharmacogenomics into clinical practice could allow providers to make optimal and informed decisions about drug selection, dose modifications, and treatment options. The ultimate goals of PGx are to individualize medicine and improve patient treatment outcomes by minimizing the risk of adverse drug events. Pharmacogenomic ushers a new era in targeted therapy to reduce the risks associated with the trial-error prescribing strategies. Moreover, pharmacogenomics could improve adherence to treatment by identifying optimal responders or those at risk for drug toxicity. ${ }^{1}$ Adherence to urate-lowering therapy (ULT) is a challenge despite published guidelines and evidence-based recommendations, urging the need for patient-centered treatment decisions. In a large longitudinal study, patients with gout achieved the lowest adherence rates to ULT relative to patients with six common diseases. ${ }^{2}$ To this end, the role of pharmacogenomics in avoiding trial-error prescribing 
and improving adherence to ULT is worth exploring. ${ }^{3}$ Additionally, gout patients receive several long-term medications, which increases their pill burden and the risk of drug-drug interactions. We believe that the PGx could help determine those who are more or less likely to derive benefits from treatments and ultimately improve the already existing poor adherence.

\section{Epidemiology of Hyperuricemia and Gout}

Gout is an inflammatory disease and the most common form of arthritis. Gout disease affects 3.9\% (9.2 million) of the United States adults. ${ }^{4}$ The prevalence of gout among individuals of African American, European Whites (EUR), and Hispanic descent was estimated to be $4.8 \%, 4 \%$, and $\%$, respectively. Notably, Asian Americans are 2.7-fold more likely to be diagnosed with gout than EUR. ${ }^{5}$ Considering the high risk of gout in Asian subgroups, Filipino Americans (FA) males have a 2.5\% incidence of gout, which is higher than the incidence in non-FA $(0.13 \%) .{ }^{5}$ High uric acid, the precursor to gout, may accumulate in articular and non-articular tissue structures, forming the monosodium urate (MSU) crystals. ${ }^{4}$ Urate underexcretion of urate is considered the predominant pathogenesis of hyperuricemia. The increased dietary intake of purine-rich sources, endogenous cell turnover, and decreased extrarenal elimination of serum uric acid (SUA) can also contribute to the pathogenesis of high urate levels. Multiple risk factors associated with hyperuricemia include diet, comorbid diseases, certain drugs, and genetics. ${ }^{4,6}$ Consistent with the contribution of genetics and risk of gout or hyperuricemia (HU), ${ }^{5}$ specific gout-related genes were significantly more prevalent in Japanese (JPT) followed by Han-Chinese (CHS) compared to EUR (p-value: <0.005). ${ }^{5}$

Patients with gout may present with acute inflammatory arthritis, subcutaneous accumulation of MSU crystals (i.e., tophi), joint damage, and chronic gouty arthritis. ${ }^{4}$ Other non-articular clinical features, such as renal or kidney stones, may also result from HU.7 Further, HU has been significantly associated with the incidence of hypertension (HTN) in adults aged $\geq 40$ years. ${ }^{8}$ Additionally, it is associated with a $20 \%$ increased prevalence of HTN, ${ }^{9}$, and a higher risk of insulin resistance. ${ }^{10}$ Based on those observations, reducing urate levels has become an important therapeutic target to treat gout and potentially manage other comorbid conditions.

\section{Genetics of Hyperuricemia and Gout}

Two-thirds of SUA is eliminated through the renal proximal tubule (RPT), while the remaining one-third is eliminated through the small intestine and metabolized by the gut microflora. ${ }^{11}$ Approximately, $90 \%$ of UA processed by the kidney is reabsorbed through the proximal tubular cells. ${ }^{12}$ Though several aspects of UA elimination and reabsorption remain unknown, extensive population genetic studies, particularly genome-wide association studies (GWAS), have identified key genetic polymorphisms in the UA disposition pathway. ${ }^{12}$ Variations in genes regulating UA excretion (ABCG2, SLC17A1), UA reabsorption (SLC22A12, SLC2A9, and SLC22A11), and a lipid metabolizing gene (GCKR), as well as a scaffolding protein (PDZK1) have all been linked to SUA levels. ${ }^{12}$

These major urate transporter proteins encoded by the above genes are involved in various functions in the UA disposition pathway. For instance, the solute carrier family 22 member 12 (SLC22A12) encodes the kidney-specific urate transporter URAT1, which is found on the apical surface of the renal proximal tubule epithelial cells. ${ }^{13}$ Secondly, the apical ATP-binding cassette transporter G2 (ABCG2) (i.e., breast cancer resistance protein) is involved in urate excretion into the distal renal tubule. ${ }^{14,15}$ Thirdly, the key player in transporting UA into the interstitial space and circulation is the GLUT9 (SLC2A9). ${ }^{16,17}$ Other transporters identified in GWAS, including OAT1, OAT3, and OAT4, are thought to play minor roles in the urate transpotome. ${ }^{14}$ Considering the inhibition of urate reabsorption as a therapeutic target in managing gout, the interplay between the single nucleotide polymorphisms (SNPs) within these transporters and the uricosuric 
pharmacotherapies further highlight the potential of pharmacogenomics to guide and personalize drug therapy in patients with gout and hyperuricemia.

\section{Methods}

The purpose of this narrative review is to provide a contemporary evaluation of pharmacogenomics in gout-related treatments. Our method involved a literature search of PubMed using the search terms ["pharmacogenomics" AND "gout"]. Titles and abstracts were scanned for relevant articles. References for selected studies were scanned for the inclusion of additional relevant articles. The primary objective of this narrative review is to synthesize the most contemporary levels of evidence of pharmacogenomics in gout pharmacotherapy. Additionally, we used the CPIC guidelines, Food and Drug Administration (FDA), and Pharmacogenetic Knowledge Base (PharmGKB) to assess the level of evidence for testing specific drug-gene pairs.

\section{Gout Management Pharmacotherapy}

The pharmacotherapy management of gout includes rapid and effective control of the inflammation in acute gout flares, continued ULT to prevent future flares, and ultimately improve gout treatment outcomes. ${ }^{4}$ Contemporary gout treatment guidelines recommend allopurinol as the preferred first-line for managing chronic gout. Pharmacotherapies, such as nonsteroidal anti-inflammatories (NSAIDs), colchicine, and corticosteroids, are also appropriate first-line agents utilized to manage gout flares. ${ }^{4}$ Pharmacotherapies, including interleukin-1 inhibitors (i.e., canakinumab and rilonacept), are also used to control gout flares when alternatives are contraindicated or ineffective. Social and environmental factors, as well as diet and genetics, could affect a patient's adherence and response to ULTs. As the field of pharmacogenomics continues to evolve, multiple studies have evaluated the effect of gene variants, including G6PD, HLA-B*58:01, and CYP2C9, on predicting the response to ULTs and possible adverse drug reactions. ${ }^{18,19}$ (Table $\mathbf{1}$ ) 
Table-1: Pharmacogenomics Summary of Gout Treatment and CPIC Guideline Level of Evidence

\begin{tabular}{|c|c|c|c|c|c|}
\hline Drug & Gene/SNP & Purpose & Impact/Outcome/Phenotype & $\begin{array}{c}\text { CPIC } \\
\text { Guideline } \\
\text { Level of } \\
\text { Evidence a }\end{array}$ & References \\
\hline \multicolumn{6}{|c|}{ Xanthine oxidase inhibitor (XO) } \\
\hline \multirow{4}{*}{$\begin{array}{l}\text { Allopurinol or } \\
\text { Oxypurinol }\end{array}$} & $H L A-B$ & Safety & $\begin{array}{l}H L A-B * 58: 01 \text { variant significantly increases risk of allopurinol-induced } \\
\text { serious cutaneous reaction }\end{array}$ & A & 1,2 \\
\hline & $A O X$ & Response & $\begin{array}{c}\text { SNP rs3731722 } \mathrm{A}>\mathrm{G} \text { is associated with better response to standard dose of } \\
\text { allopurinol ( } 300 \mathrm{mg} / \text { day) vs. non-carriers }\end{array}$ & NA & 3 \\
\hline & $A B C G 2$ & Response/PK & SNP rs2231142 C>A in this gene is associated with poor response. & NA & 4 \\
\hline & $S L C 22 A 12$ & Response/PK & $\begin{array}{c}\text { SNP rs505802 C }>\text { T may potentially influence response to allopurinol as } \\
\text { oxypurinol is a substrate for the URAT1 }\end{array}$ & NA & $5-7$ \\
\hline Febuxostat & $\begin{array}{l}\text { UGT1 member } A \\
\text { cluster }\end{array}$ & Response/PK & SNP rs34650714 C $>\mathrm{T}$ is associated with lower doses of febuxostat. & NA & 3 \\
\hline \multicolumn{6}{|l|}{ Uricosuric } \\
\hline \multirow{3}{*}{ Probenecid } & $S L C 22 A 12$ & Response & $\begin{array}{l}\text { Homozygous or heterozygous for the mutant allele (G774A) has impaired response to } \\
\text { loading tests. }\end{array}$ & NA & 8,9 \\
\hline & $A B C B 1$ & PK & $\begin{array}{l}\text { SNP (rs1045642 C }>\text { T) influence the PK effect of probenecid as an inhibitor } \\
\text { when co-administered with Beta-lactam }\end{array}$ & NA & 10 \\
\hline & $G 6 P D$ & Safety & Possible hematologic adverse reactions in G6PD deficient. & $\mathrm{B}$ & 11 \\
\hline \multicolumn{6}{|c|}{ Recombinant Uricase } \\
\hline Pegloticase & $G 6 P D$ & Safety & Risk of hemolysis or methemoglobinemia in G6PD deficient. & $\mathrm{B}$ & 12 \\
\hline \multicolumn{6}{|c|}{ Non-steroidal anti-inflammatory drugs (NSAIDs) } \\
\hline
\end{tabular}

Non-steroidal anti-inflammatory drugs (NSAIDs) 


\begin{tabular}{|c|c|c|c|c|c|}
\hline $\begin{array}{l}\text { Ibuprofen, } \\
\text { celecoxib, and } \\
\text { other NSAIDs }\end{array}$ & CYP2C9 & Safety/PK & $\begin{array}{l}\text { Increased risk of NSAID-related GI bleeding in no-function allele }(* 3) \text { carriers } \\
\text { relative to normal function, as well as reduced metabolism and prolonged } \\
\text { exposure to ibuprofen and celecoxib in poor metabolizers. }\end{array}$ & $\begin{array}{l}\text { A (ibuprofen } \\
\text { and } \\
\text { celecoxib); C } \\
\text { (indomethacin } \\
\text {, diclofenac, } \\
\text { naproxen) }\end{array}$ & 13,14 \\
\hline \multicolumn{6}{|c|}{ Anti-inflammatory } \\
\hline \multirow{4}{*}{ Colchicine } & CYP2D6 & \multirow[b]{2}{*}{ Response } & Diminished response in $C Y P 2 D 6^{*} 4$ variant carriers. & NA & 15 \\
\hline & $A B C B 1$ & & $\begin{array}{l}\text { Inconsistent evidence where one indicates nonresponse in major allele carriers } \\
\text { of the SNP rs } 10455642 \mathrm{C}>\mathrm{T} \text {, while another suggests relationship of T allele in } \\
\text { same SNP to nonresponse. }\end{array}$ & NA & 16,17 \\
\hline & SEPHS1 & Safety & $\begin{array}{l}\text { The risk allele G vs. A, at SNP rs } 74795203 \mathrm{~T}>\mathrm{G} \text { significantly increases risk of } \\
\text { gastrointestinal adverse events by } 2.5 \text {-fold when the drug used. }\end{array}$ & NA & 18 \\
\hline & $\begin{array}{c}\text { rs6916345 G>A } \\
\text { located on } \\
\text { chromosome } 6 \\
\text { locus }\end{array}$ & Safety & $\begin{array}{l}\text { The risk allele A significantly increases risk of gastrointestinal adverse events } \\
\text { by } \sim 2 \text {-fold when the drug used compared with the G allele. }\end{array}$ & NA & 18 \\
\hline \multicolumn{6}{|l|}{ Corticosteroids } \\
\hline $\begin{array}{l}\text { Injectable } \\
\text { triamcinolone } \\
\text { acetonide }\end{array}$ & HCG22 & Safety & $\begin{array}{l}\text { The } \mathrm{G} \text { and } \mathrm{T} \text { alleles of SNPs rs } 3873352 \mathrm{C}>\mathrm{G} \text { and rs } 2523864 \mathrm{C}>\mathrm{T} \text {, } \\
\text { respectively, increases risk of steroid-induced ocular hypertension. }\end{array}$ & NA & 19 \\
\hline \multicolumn{6}{|l|}{ IL-1 inhibitor } \\
\hline Anakinra & $I L 1 R N$ & Response & $\begin{array}{l}\text { SNP cluster in strong linkage disequilibrium is associated with poor } \\
\text { response. }\end{array}$ & NA & 20 \\
\hline
\end{tabular}

Table-1 Legend: PK, Pharmacokinetic; SNP, Single nucleotide polymorphism; CPIC Clinical Pharmacogenomic Implementation Consortium; HLA-B, Human leukocyte antigen B; $A O X$, Aldehyde oxidase; $A B C G 2$, ATP-binding cassette transporter G2; UGT1 (members $A 1$ and $A 3-10$ ), Uridine diphosphate (UDP) glucoronosyl-transferase family 1; $S L C 22 A 12$, Solute carrier family 22 member 12; $A B C B 1$, Human adenosine triphosphate (ATP)-binding cassette subfamily B member 1; G6PD, Glucose-6-phosphate- 
dehydrogenase; CYP2C9, Cytochrome P450 2C9; CYP2D6, Cytochrome P450 2D6; SEPHS1, selenophosphate synthase 1; HCG22, HLA complex group 22; IL-1RN, Interleukin-1 receptor antagonist.

${ }^{\text {a }} \mathrm{CPIC}$ level in the clinical context for rapid interpretation by clinicians includes A: Genetic information should be used to change prescribing of affected drug (quality of evidence is high and in favor of changing prescribing); B: genetic information could be used to change prescribing of the affected drug, but alternative drugs are as effective and safe as nongenetically based dosing (optional change in prescribing); C: evidence levels varies and no prescribing actions recommended; and D: evidence is weak and conflicting; and no prescribing actions recommended.

\section{Reference:}

1. Saito Y, Stamp LK, Caudle KE, et al. Clinical Pharmacogenetics Implementation Consortium (CPIC) guidelines for human leukocyte antigen B (HLA-B) genotype and allopurinol dosing: 2015 update. Clinical pharmacology and therapeutics. 2016;99(1):36-37. doi:10.1002/CPT.161

2. FitzGerald JD, Dalbeth N, Mikuls T, et al. 2020 American College of Rheumatology Guideline for the Management of Gout. Arthritis Care and Research. 2020;72(6):744760. doi:10.1002/ACR.24180/ABSTRACT)

3. Carroll MB, Smith DM, Shaak TL. Genomic sequencing of uric acid metabolizing and clearing genes in relationship to xanthine oxidase inhibitor dose. Rheumatology international. 2017;37(3):445-453. doi:10.1007/S00296-016-3592-2

4. Vora B, Brackman DJ, Zou L, et al. Oxypurinol pharmacokinetics and pharmacodynamics in healthy volunteers: Influence of BCRP Q141K polymorphism and patient characteristics. Clinical and translational science. 2021;14(4):1431-1443. doi:10.1111/CTS.12992

5. Iwanaga T, Kobayashi D, Hirayama M, Maeda T, Tamai I. Involvement of uric acid transporter in increased renal clearance of the xanthine oxidase inhibitor oxypurinol induced by a uricosuric agent, benzbromarone. Drug metabolism and disposition: the biological fate of chemicals. 2005;33(12):1791-1795. doi:10.1124/DMD.105.006056

6. Roman YM, Culhane-Pera KA, Menk J, Straka RJ. Assessment of genetic polymorphisms associated with hyperuricemia or gout in the Hmong. Personalized medicine. 2016;13(5):429-440. doi:10.2217/PME-2016-0021

7. Alghubayshi A, Edelman A, Alrajeh K, Roman Y. Genetic assessment of hyperuricemia and gout in Asian, Native Hawaiian, and Pacific Islander subgroups of pregnant women: biospecimens repository cross-sectional study. BMC rheumatology. 2022;6(1). doi:10.1186/S41927-021-00239-7

8. Ichida K, Hosoyamada M, Hisatome I, et al. Clinical and molecular analysis of patients with renal hypouricemia in Japan-influence of URAT1 gene on urinary urate excretion. Journal of the American Society of Nephrology: JASN. 2004;15(1):164-173. doi:10.1097/01.ASN.0000105320.04395.D0

9. Hamada T, Ichida K, Hosoyamada M, et al. Uricosuric action of losartan via the inhibition of urate transporter 1 (URAT 1) in hypertensive patients. American journal of hypertension. 2008;21(10):1157-1162. doi:10.1038/AJH.2008.245

10. Beringer PM, Kriengkauykiat J, Zhang X, et al. Lack of effect of P-glycoprotein inhibition on renal clearance of dicloxacillin in patients with cystic fibrosis. Pharmacotherapy. 2008;28(7):883-894. doi:10.1592/PHCO.28.7.883 
11. Chan TK, Todd D, Tso SC. Drug-induced haemolysis in glucose-6-phosphate dehydrogenase deficiency. British Medical Journal. 1976;2(6046):1227. doi:10.1136/BMJ.2.6046.1227

12. Relling M v., Mcdonagh EM, Chang T, et al. Clinical Pharmacogenetics Implementation Consortium (CPIC) guidelines for rasburicase therapy in the context of G6PD deficiency genotype. Clinical pharmacology and therapeutics. 2014;96(2):169-174. doi:10.1038/CLPT.2014.97

13. Theken KN, Lee CR, Gong L, et al. Clinical Pharmacogenetics Implementation Consortium Guideline (CPIC) for CYP2C9 and Nonsteroidal Anti-Inflammatory Drugs. CLINICAL PHARMACOLOGY \& THERAPEUTICS I. doi:10.1002/cpt.1830

14. Figueiras A, Estany-Gestal A, Aguirre C, et al. CYP2C9 variants as a risk modifier of NSAID-related gastrointestinal bleeding: a case-control study. Pharmacogenetics and Genomics. 2016;26(2):66. doi:10.1097/FPC.0000000000000186

15. Yalcintepe S, Ozdemir O, Silan C, et al. The CYP4502D6 ${ }^{*} 4$ and ${ }^{*} 6$ alleles are the molecular genetic markers for drug response: implications in colchicine nonresponder FMF patients. European journal of drug metabolism and pharmacokinetics. 2016;41(3):281-286. doi:10.1007/S13318-015-0255-8

16. Ozen F, Silan C, Uludag A, et al. Association between ABCB1 (MDR1) gene $3435 \mathrm{C}>\mathrm{T}$ polymorphism and colchicine unresponsiveness of FMF patients. Renal failure. 2011;33(9):899-903. doi:10.3109/0886022X.2011.605980

17. Babaoglu MO, Yasar U, Tufan A, et al. Association of the $3435 \mathrm{C}>\mathrm{T}$ polymorphism of the drug transporter gene ABCB1 with colchicine response in patients with familial Mediterranean fever . The FASEB Journal. 2007;21(5). doi:10.1096/FASEBJ.21.5.A414-D

18. Dubé MP, Legault MA, Lemaçon A, et al. Pharmacogenomics of the Efficacy and Safety of Colchicine in COLCOT. Circulation Genomic and precision medicine. 2021;14(2):223-229. doi:10.1161/CIRCGEN.120.003183

19. Jeong S, Patel N, Edlund CK, et al. Identification of a Novel Mucin Gene HCG22 Associated With Steroid-Induced Ocular Hypertension. Investigative ophthalmology E visual science. 2015;56(4):2737-2748. doi:10.1167/IOVS.14-14803

20. Pardeo M, Rossi MN, Pires Marafon D, et al. Early Treatment and IL1RN Single-Nucleotide Polymorphisms Affect Response to Anakinra in Systemic Juvenile Idiopathic Arthritis. Arthritis \& rheumatology (Hoboken, NJ). 2021;73(6):1053-1061. doi:10.1002/ART.41612 


\section{Allopurinol}

The purine analog and xanthine oxidase $(\mathrm{XO})$ inhibitor, allopurinol, is widely utilized to manage hyperuricemia and gout. ${ }^{4}$ Allopurinol inhibits the conversion of hypoxanthine to xanthine, mediated by $\mathrm{XO}$, ultimately reducing uric acid production. ${ }^{20}$ Allopurinol is metabolized to oxypurinol, the active metabolite of allopurinol. Oxypruinol and allopurinol are known substrates for the efflux transporter, so-called breast cancer resistant protein (BCRP). Hence, multiple studies investigated the interplay between BCRP protein and the intestinal disposition of allopurinol and renal elimination of oxypurinol. ${ }^{21}$

Allopurinol is generally safe; however, it is associated with the risk of a life-threatening syndrome characterized as eosinophilia and serious cutaneous adverse reaction (SCAR), so-called allopurinol-induced hypersensitivity syndrome (AHS), in specific patient populations. ${ }^{22,23}$ Ethnicity, renal impairment, diuretic use, allopurinol starting dose, and the presence of $H L A-B^{*} 5801$ are risk factors that contribute to the development of AHS.22-24 The human leukocyte antigen B (HLA-B), part of the Major Histocompatibility Complex-I (MHC-I) is considered the most polymorphic gene in the human genome, with more than 1500 alleles. ${ }^{22}$ Carriers of the $H L A-B^{*}$ 58:01 variant are at greater risk for SCARs than non-carriers. Thus, the use of allopurinol in patients carrying $H L A-B * 58: 01$ is contraindicated. ${ }^{25}$ The latter is associated with an 80-97-fold increased risk of allopurinol-induced hypersensitivity syndrome. ${ }^{26}$

$H L A-B^{*}$ 58:01 prevalence is markedly higher in African Americans and Asian subpopulations, mainly Korean, Han Chinese, and Thai descents, which substantiates the recommendation for $H L A-B^{*} 5801$ genotyping in these populations. ${ }^{18}$ Nonetheless, the United States Food and Drug Administration (FDA) did not include PGx recommendations on the drug label. However, it listed the drug in the pharmacogenetic association table. ${ }^{27}$ The CPIC and the American College of Rheumatology (ACR) provide clinical guidance on pharmacogenetic testing for $H L A-B^{*} 58: 01$. Collectively, it is highly recommended to test for $H L A-B^{*} 58: 01$ before commencing allopurinol among high-risk populations to provide alternative therapies in the allele carriers. ${ }^{18,28}$

Oxypurinol levels have been used to measure allopurinol adherence and drug-therapeutic monitoring among patients with gout. Thus, genetic polymorphisms in the allopurinol-metabolizing enzyme gene are of great interest. Theoretically, oxypurinol formation, the endpoint of allopurinol metabolism with the longest half-life, could partly explain the heterogeneity of response to allopurinol therapy. Therefore, processes involved in the formation of oxypurinol could influence active metabolite exposure and the magnitude of urate reduction. It is known that aldehyde oxidase (AOX) converts allopurinol to oxypurinol. The rs3731722 A $>$ G SNP leads to a nonsynonymous codon change in $A O X$, and it is associated with achieving SUA target with a standard dose of allopurinol (300 mg daily) relative to non-carriers (i.e., wild type allele A). ${ }^{29}$ Though the association is intriguing; it remains of low clinical significance. ${ }^{29}$ Other genes involved in the metabolism and clearance of allopurinol were also studied. Firstly, the XO protein is the therapeutic target for febuxostat and allopurinol, encoded by the XO gene. Secondly, Molybdenum cofactor sulfurase (MOCOS) incorporates molybdenum cofactor in purine oxidases (e.g., AOX and XO). Plausibly, genetic variation in the MOCOS gene may result in defective purine oxidases, altering allopurinol metabolism. ${ }^{29}$

Besides the potential impact of $H L A-B$ in predicting toxicity to allopurinol, $A B C G 2$ could also predict the response to allopurinol. ${ }^{21,30} \mathrm{~A}$ single nucleotide polymorphism (SNP) within $A B C G 2$, specifically the missense variant p.Q141K (rs2231142 C>A), results in a reduced function in the secretory transporter in the kidney and gut, affecting the pharmacokinetic (PK) and pharmacodynamic (PD) of allopurinol and its metabolite as they are substrates for the ABCG2 transporter. ${ }^{21,30}$ Paradoxically, the minor allele of rs2231142 C>A was linked to a lower reduction in SUA levels in response to allopurinol therapy in one GWAS. ${ }^{30}$ The reduced function variant of ABCG2 supposedly leads to a decreased clearance and greater systemic plasma levels of the drug and its metabolite; hence a more 
considerable SUA decrease is expected. In a prospective clinical study, utilizing realworld data, relative to the homozygous for the wild-type alleles (CC), the homozygous for the reduced function alleles (AA) had a significantly longer half-life of oxypurinol ( $t_{1 / 2}$ $19.1 \pm 1.42$ vs. $34.2 \pm 12.2$; p-value: 0.047$).{ }^{21}$

The exact mechanism by which the polymorphism induces a decreased allopurinol response is unknown. Nonetheless, some evidence suggests that allopurinol and oxypurinol likely directly affect UA reabsorption besides inhibiting its production. ${ }^{31}$ For example, in-vitro studies demonstrated that allopurinol and oxypurinol have uricosuric effects through inhibition of SUA reabsorption via competitive inhibition of URAT1 ${ }^{32}$ or potent inhibition of GLUT9, ${ }^{33}$ known SUA reabsorption transporters. Further research, particularly the analysis of uncommon polymorphisms in $A B C G 2$, will undoubtedly improve our knowledge of the genetic basis of the response to allopurinol.

URAT1, encoded by SLC22A12, is considered the predominant transporter for UA reabsorption in the kidney. Presumably, a gain-of-function polymorphism in URAT1 could result in high SUA, whereas loss-of-function mutations cause idiopathic renal hypouricemia due to high urinary urate clearance. ${ }^{34}$ The $\mathrm{T}$ allele of the intergenic SNP rs505802 C>T in SLC22A12 was linked with lower SUA than the allele (C). The prevalence of the T allele was significantly low (p-value: $<0.01)$ in Asian $(18-35 \%)$, Native Hawaiian, and Pacific Islanders (5-37\%), relative to EUR ( 72\%). ${ }^{34,35}$ The disparity in the prevalence of the $\mathrm{T}$ allele may not only indicate that Asian and Pacific Islanders populations are more prone to hyperuricemia relative to EUR, but also provides the basis for inquiring about the response to allopurinol and oxypurinol among the same populations. ${ }^{34}$ In-vitro study suggested that the inhibition of URAT1 by benzbromarone facilitated renal clearance of the active metabolite of allopurinol. ${ }^{32}$

While genetics are important covariates, sex and ABCG2 inhibitors (e.g., cyclosporin) may also influence the response to allopurinol. ${ }^{21}$ The volume of distribution (Vd) of oxypurinol in females is $0.248 \mathrm{~L}$ lower than in males, which is significant (95\% CI: -0.395, 0.067). ${ }^{21}$ The low Vd in women compared to men could plausibly translate to a lower dose requirement of the drug to achieve the target plasma concentration. In a retrospective cohort study, the mean dose difference of allopurinol at the time of reaching target SUA (< $6.5 \mathrm{mg} / \mathrm{dL}$ ) was significantly lower in women $(216 \mathrm{mg})$ compared to men $(217 \mathrm{mg})$ (difference: $-55 \mathrm{mg}$; $95 \% \mathrm{CI}$ : $-73,-37) ;{ }^{36}$ however, sex did not demonstrate differences in the overall response to allopurinol after six months of follow up period in the same study. ${ }^{36}$

\section{Febuxostat}

Febuxostat is structurally unrelated to purine and a potent $\mathrm{XO}$ inhibitor. ${ }^{20}$ Febuxostat has FDA approval for the chronic management of hyperuricemia in patients with gout. ${ }^{37}$ Although it is well-tolerated at the recommended dose, it is associated with a higher prevalence of abnormal hepatic enzymes than allopurinol. ${ }^{20,38}$ Additionally, an inconsistent body of evidence suggests that febuxostat is associated with an increased risk of death from cardiovascular disease (CVD). Notably, febuxostat users are 34\% more likely to have CVD-related mortality and $22 \%$ all-cause mortalities than allopurinol. Hence, the conditional recommendation to switch to an alternative ULT in patients with a history of CVD or a new CVD-related event. ${ }^{4,39}$ However, recent studies found no association between febuxostat and CVD or all-cause mortality. ${ }^{40,41}$ -

Patients who utilize $\mathrm{XO}$ inhibitors require varying doses to achieve an appropriate SUA target. It is reasonable to assume that some of those variations are due to polymorphisms in metabolism and clearance genes of XO inhibitors. ${ }^{29}$ The uridine diphosphate (UDP) glucoronosyl-transferase (UGT) enzyme metabolizes febuxostat by conjugation (i.e., glucuronidation). ${ }^{20,38}$ The $U G T$ family 1 member $A$ cluster (i.e., members $A 1$, and $A 3$ to 10) gene encodes UGT protein. ${ }^{20,29}$ UGT1A1 is considered clinically actionable druggene or very important pharmcogenes (VIP). ${ }^{42}$ A single-nucleotide polymorphism (SNP), rs34650714 C>T, in the UGT1A1 appears to be associated with lower febuxostat dose requirement, dose lower than $300 \mathrm{mg}$ daily equivalent to allopurinol, relative to the wild- 
type $C$ allele in patients with gout (p-value: 0.007 ). However, this SNP is an intronic variant in a non-amino acid coding region. ${ }^{29}$ Further, the nonsynonymous change from $\mathrm{A}$ to $\mathrm{G}$ at the rs28898617 of UGT1A4 is associated with a difference of $5.1 \mathrm{mg} / \mathrm{dL}$ in mean SUA versus wild type allele. ${ }^{29}$ These findings reflect the importance of phase II drug-metabolizing encoding genes (UGT1A1 and UGT1A4) and thus may influence the response to XO inhibitors. ${ }^{29}$

\section{Uricosurics}

The management of hyperuricemia could also be mediated by increasing excretion or inhibiting reabsorption of urate through the kidney. Uricosuric drugs, including probenecid, benzbromarone, and lesinurad, are used as a second line to manage gout and hyperuricemia. Most uricosuric pharmacotherapies block URAT1 transporter, preventing renal UA reabsorption. ${ }^{14,43}$ Besides URAT1, probenecid inhibits other transporters, including OAT1, OAT3, and GLUT9.43 Several studies demonstrated the interplay between genetic polymorphisms within urate transporters, the pathology of hyperuricemia, and the clinical response to uricosuric drugs. ${ }^{44,45}$

In patients with renal hyperuricemia, the $774 \mathrm{G}>\mathrm{A}$ mutation carriers in SLC22A12 had significantly lower SUA concentration and higher urate clearance rates relative to healthy control. ${ }^{4}$ Notably, carriers of homozygous or heterozygous for the mutant allele (G774A) had impaired response to loading tests with probenecid and benzbromarone. ${ }^{44}$ Following the previous study results, Hamada et al. ${ }^{45}$ studied the effect of loss-of-function SLC22A12 mutations on urate clearance rates using benzbromarone and losartan in patients with idiopathic hyperuricemia and hypertension. Neither losartan nor benzbromarone affected urate clearance in patients carrying mutant SLC22A12 (G774A). Conversely, when either drug was used, the wild-type SLC22A12 had significantly increased urate clearance ( 7 to $13 \%$ with benzbromarone; $p$-value $<0.01$ ). ${ }^{45}$ These experimental observations provide evidence that genetic mutations within SLC22A12 are also of PGx relevance.

The ABCB1 (P-glycoprotein), the adenosine triphosphate (ATP)-binding cassette subfamily $B$ member 1 , is an efflux transporter of various drugs. It is essential for the disposition and effects of multiple drugs. The highly polymorphic gene, $A B C B 1$, codes for the same transporter and is considered an important pharmacogenetic biomarker with clinical relevance. ${ }^{46}$ The induction or inhibition of ABCB1 by a drug (i.e., an underlying mechanism of drug-drug-transporter interactions) is a critical determinant of the PK profile of the affected drug. A PK randomized-controlled study demonstrates the implication of a drug-drug-ABCB1 interaction and the possibility of genetic influence. The investigators used probenecid to determine the role of ABCB1 in explaining the increased excretion of dicloxacillin transport in cystic fibrosis patients. ${ }^{47}$

When administered with probenecid, clearance and distribution of dicloxacillin decreased, implying that probenecid is an inhibitor of ABCB1 and the latter is a substrate for the same transporter. ${ }^{47}$ On the contrary, dicloxacillin urinary excretion was significantly higher when administered with probenecid in patients with CC genotype of the $A B C B 1$ (i.e., rs1045642 C>T) than CT (p-value $<0.01$ ). While this phenomenon highlights the point that there is a possible genetic effect on probenecid efficacy as an inhibitor of ABCB1, extensive studies are needed to discern this and confirm their applicability to gout patients. ${ }^{47}$

The probenecid label indicates that risks of hematologic ADRs (i.e., aplastic, hemolytic anemia, or leukopenia) are associated with probenecid administration in patients with glucose-6-phosphate-dehydrogenase (G6PD) deficiency. However, this annotation was listed under the ADRs list, and there were no PGx recommendations. ${ }^{48} \mathrm{~A}$ small cohort study found that the G6PD deficiency was not associated with hemolysis when probenecid was used in East Asians. ${ }^{49}$ The evidence indicates that genetic polymorphisms in efficacy and safety genes may influence PK/PD and toxicity profiles of uricosurics, though additional studies are warranted to support preemptive pharmacogenetic testing.

\section{Recombinant Uricases}


Rasburicase and pegloticase are recombinant forms of the uricase enzyme. Higher hominoids (apes and humans)lost the uricase gene millions of years ago. In lower primates, uricase converts plasma uric acid to allantoin. The latter is more soluble than uric acid and readily excreted. ${ }^{50,51}$ Rasburicase is mainly used for the prevention and treatment of hyperuricemia. However, it is not suitable for chronic gout treatment due to its limited pharmacokinetics parameters (short half-life and fast-acting). Besides, it is more effective in reducing plasma uric acid levels in cancer patients than allopurinol in tumor lysis syndrome management. ${ }^{52,53}$ Unlike rasburicase, pegloticase is more suitable and commonly used to treat chronic gout.

G6PD transforms glucose-6-phosphate to 6-phosphogluconolactone, resulting in the production of reduced nicotinamide adenine dinucleotide phosphate (NADPH) from nicotinamide dinucleotide phosphate. G6PD is fundamental in erythrocyte integrity because G6PD and 6-phosphogluconate dehydrogenase (6-PD) are the primary sources of NADPH. The latter protects the red blood cells (RBCs) from oxidative stress, such as oxygen radicals or hydrogen peroxide. While oxidative stress may occur as a result of abnormal physiological changes, it could be induced by exposure to specific drug therapy. ${ }^{54}$

G6PD-deficient RBCs, for example, significantly decrease NADPH production capacity, and hence, they are more susceptible to hemolytic anemia (AHA). Moreover, the oxidation of hemoglobin iron results in the formation of methemoglobin, which cannot carry oxygen or carbon dioxide. This condition is known as methemoglobinemia (MGM), which is characterized by cyanosis and, in severe cases, may lead to arrhythmias, seizures, and death. 55

Pegloticase is associated with an increased risk of developing MGM and AHA in some patients. These significant drug adverse effects could originate from reactive oxygen species formation due to urate oxidation to allantoin. Notably, MGM and AHA are strongly linked to the activity levels in G6PD. Notably, specific genetic polymorphisms in G6PD may result in different activity levels and hence modulate the risk of developing MGM or AHA. Therefore, genetic testing of G6PD, especially in specific patient populations (i.e., African, South European, Middle Eastern, South Asian), could garner improved outcomes before initiating pegloticase therapy. ${ }^{55}$

Similar to PEG, rasburicase is contraindicated in patients with G6PD deficiency. Rasburicase administration may also lead to oxidative damage to the red blood cells. However, this risk is only limited to patients who are G6PD-deficient. G6PD-deficient is characterized as a male who has one allele of the World Health Organization (WHO) class-II or -III, or a female who carries two alleles (e.g., class II-III variants "II/II, III/III, or II/III"). ${ }^{56}$ The prevalence of the G6PD-deficiency varies between different populations with a marked increase in African (i.e., Sub-Saharan African countries) or Mediterranean ancestry patients. ${ }^{57}$ Therefore, a prompted genotype testing for the G6PD gene is advised in these patients. ${ }^{51}$

Also, the FDA issued a Blackbox warning for rasburicase and pegloticase due to the risk of hemolysis or methemoglobinemia in patients with G6PD deficiency (i.e., $<10-60 \%$ of normal enzyme activity). Although uncommon $(<1 \%)$, these adverse events are significantly severe and could be life-threatening. ${ }^{29}$

\section{NSAIDs}

Non-steroidal anti-inflammatory drugs (NSAIDs) have been extensively used to manage various disease conditions, including acute gout flares, due to their anti-inflammatory and pain-relieving mechanism of action. NSAIDs inhibit the conversion of arachidonic acid to prostaglandins, involved in pain signaling cascade during inflammation, inhibiting the cyclooxygenase (COX) enzyme. Thus, targeting the prostaglandins and inhibiting the inflammatory cascade is a reasonable mechanism to reduce gout flares-associated pain. ${ }^{58}$

While NSAIDs are efficacious and less costly medications to manage gout pain, prescribers need to balance between the different risks associated with various NSAIDs and 
the use of concomitant drugs that would potentiate these risks. NSAID adverse reactions, including cardiovascular (CVD) events, gastrointestinal (GI) bleeding, and renal impairment, are essential to consider before selecting any of them. ${ }^{58}$

Most NSAIDs (e.g., indomethacin, ibuprofen, naproxen, diclofenac, meloxicam, and celecoxib) are substrates for the hepatic metabolism via CYP2C9, 1A2, and 3A4 enzymes. Also, the majority of NSAIDs are renally excreted. ${ }^{8}$ The CYP2C9 is a phase-I cytochrome P450 drug-metabolizing enzyme isoform involved in oxidizing endogenous and exogenous compounds, including NSAIDs. The gene coding for the CYP2C9 is highly polymorphic with more than 70 known variants. ${ }^{59}$ Variations in the CYP2C9 gene could result in varying drug-metabolizing ability. Hence, the predicted metabolizer phenotypes, based on the individual's CYP2C9 genotype, are normal (NM), extensive (EM), intermediate (IM), and poor (PM) metabolizers. ${ }^{60,61}$ Nonetheless, the enzymatic activity also depends on the individual's age, sex, comorbid diseases, and concomitant use of certain medications (i.e., other CYP2C9 substrates, inhibitors, or inducers). ${ }^{62}$ Furthermore, variability in the activity of CYP2C9 impacts the exposure to NSAIDs. ${ }^{62}$

Importantly, the $C Y P 2 C 9 * 2$ (i.e., decreased function) variant is in strong linkage disequilibrium (LD) with the $C Y P 2 C 8 * 3$ allele. This LD might have therapeutic implications for medications like diclofenac and ibuprofen, which are both CYP2C9 and CYP2C8 substrates. $^{62}$

A case-control study found that the odds of NSAID-related upper GI bleeding were 16.92 (95\% CI 4.96-57.59) in CYP2C9*3 (i.e., no function allele) carriers and NSAID users (with higher average maintenance daily dose), compared to non-carriers 9.72 (95\% CI 4.5520.76). ${ }^{63}$ In addition, the CPIC guidelines provide CYP2C9 genotype-guided recommendations on specific NSAIDs, such as ibuprofen, celecoxib, meloxicam, and piroxicam, to reduce the toxicity risk associated with their use. ${ }^{64}$

Patients with CYP2C9 PM phenotype (i.e., ${ }^{*} 3 /{ }^{*} 3$ or ${ }^{*} 2 / 22$ diplotypes) have a substantially reduced metabolism and prolonged exposure to ibuprofen and celecoxib, thus increasing the likelihood of severe adverse outcomes. Unlike the NM and IM, individuals with the PM phenotype are permitted to start with only a quarter to half of the recommended starting dose. ${ }^{62}$

The evidence linking the CYP2C9 genetic variants to indomethacin, diclofenac, and naproxen pharmacokinetics is not significant in vivo and/or not sufficient to warrant a recommendation to guide their use in practice by the CPIC guidelines. ${ }^{62}$

The GI and CVD adverse outcomes associated with NSAIDs may have considerable public health consequences due to the vast population exposed to them. Moreover, an individualized approach that considers risk factors including genetic predisposition, older age, concomitant drug use, or pre-existing disease could be implicated before NSAID use. ${ }^{62}$ Hence, knowledge of the CYP2C9 genetic status of a patient with gout may further guide the selection between different NSAIDs or adjust the dose to minimize GI bleeding risk.

\section{Colchicine}

Colchicine is an alkaloid derived from meadow saffron. It relieves pain by inhibiting the inflammation response and the infiltration of the immunological cells at the site where MSU crystals deposit. Colchicine interferes with neutrophil functions, lysosomal degradation, and leukocyte chemotaxis by disrupting the microtubule's polymerization and mobilization. Additionally, colchicine halts the MSU-induced inflammasome-driven caspase-I activation and interleukin-1 (IL-1) beta processing. ${ }^{65}$ The most prominent adverse effects associated with colchicine are gastrointestinal (GI) adverse events, including diarrhea, nausea, and vomiting. ${ }^{66}$ Probably, these ADRs are due to binding to free tubulin that hinders the fusion of autophagic vacuoles with lysosomes in smooth muscles, resulting in damage to these organs, ${ }^{67}$ or increasing GI secretions and motility. ${ }^{68}$ Other adverse effects of colchicine include myotoxicity and neuropathy. ${ }^{68}$ 
The metabolism of colchicine, besides other xenobiotics, is mediated by the enzyme cytochrome P450 2D6 (CYP2D6). ${ }^{69}$ About 40 to 65\% of colchicine is excreted renally and excreted via the efflux p-glycoprotein (P-gp) transporter. ${ }^{70}$ The drug-metabolizing enzyme CYP2D6 is encoded by the CYP2D6 gene, a highly polymorphic drug-metabolizing gene. ${ }^{69} \mathrm{CYP} 2 \mathrm{D} 6$ is responsible for the metabolism of $25 \%$ of prescribed medication; thus, it is considered one of the most critical pharmacogenes. ${ }^{71}$ PGx studies linking CYP2D6 to clinical response to colchicine in gout patients are limited; nevertheless, the role of CYP2D6 was evaluated in a Middle Eastern ethnic population taking colchicine for Familial Mediterranean Fever (FMF).

Colchicine effectively controlled pain flares in FMF, an inherited disorder of Mediterranean origin. ${ }^{72}$ However, approximately $10-20 \%$ of patients taking colchicine treatment are resistant, and some showed a diminished response due to potential genetic variations. ${ }^{73}$ Colchicine non-responder with FMF were found to have higher CYP $2 D 6^{*} 4$ allele (no function allele) frequency than responders $(0.16 \%$ versus $0.12 \%$, respectively). However, in colchicine responders, the CYP2D6 ${ }^{*} 1 /{ }^{*} 1$ (homozygous normal function allele) genotype frequency was higher versus non-responders $(80 \%$ versus $30 \%$, respectively, the p-value was not tested). ${ }^{74}$ This observation plausibly indicates that individuals with the reduced drug-metabolizing activity of CYP2D6 could not garner the anti-inflammatory benefits of colchicine.

On the on hand, the pharmacogenomic relevance of $A B C B 1$ with the response to colchicine was assessed in FMF subjects of Turkish descent in a case-control study. ${ }^{75}$ The study suggested that the odds of diminished response to colchicine in the $\mathrm{C}$ allele carriers of the $A B C B 1$ SNP rs10455642 C T, relative to the TT, were 9.7 ( $\mathrm{p}<0.001) .{ }^{75}$ On the other hand, colchicine-resistant patients were more likely to carry the T allele $(66 \%$ for TT, $59 \%$ for $\mathrm{CT}$, and $39 \%$ for $\mathrm{CC}$ ) than responders (33\% for TT, $41 \%$ for $\mathrm{CT}$, and $61 \%$ for $\mathrm{CC}$ ) in the Israeli cohort ( $\mathrm{p}$-value: 0.047$).{ }^{76}$ The conflict in these findings proposes that $A B C B 1$ polymorphism is prone to inter-ethnic variations. ${ }^{76}$ Research on medication responsiveness, which did not include colchicine, showed inconsistent results regarding the association between alleles in $A B C B 1$ and alterations in P-gp transporter function of specific drugs in European and Asian populations. ${ }^{77}$ Although the results from the aforementioned studies might present a pharmacogenetic basis to colchicine efficacy, these findings were not corroborated in patients with gout.

Considering the anti-inflammatory effect of colchicine, a recent trial, the Colchicine Cardiovascular Outcomes Trial (COLCOT) found to be beneficial in lowering the rates of ischemic heart disease events (composite primary endpoint) by $23 \%$ in post-myocardial infarction (MI) subjects using low dose colchicine ( $0.5 \mathrm{mg}$ once daily) versus placebo (hazard ratio (HR): 0.77, p-value: 0.02). ${ }^{78}$ Additionally, a GWAS of the COLCOT study identified the two most significant SNPs on chromosomes 6 and 10 associated with the GI adverse events of colchicine (secondary safety endpoint). ${ }^{79}$

The risk allele (A) of the intergenic SNP rs6916345 G>A located on chromosome 6 increased the occurrence of GI adverse events (diarrhea) in the colchicine group by $89 \%$ (HR: 1.89 , p-value: $<0.001)$ relative to the ancestral allele $(\mathrm{G}) .{ }^{79}$ The same allele was previously identified in a GWAS and found to be associated with Crohn's disease (CD) (OR: 1.07, p-value $<0.001) .{ }^{80}$ In addition, the homozygous patients with the risk allele (AA) in the intervention arm reported GI adverse events that are 2-fold higher than those reported by patients in the placebo arm (HR: 2.42 , p-value: $<0.001) .{ }^{79}$

The second SNP rs74795203 T>G identified in the COLCOT GWAS was found on chromosome 10 and located in intron 4 of SEPHS1. ${ }^{79}$ The SEPHS1, selenophosphate synthase 1, encodes for the enzyme SEPHS1 that synthesizes selenophosphate, the selenium donor, to ultimately incorporate it into selenoproteins ${ }^{81,82}$ SEPHS1 plays a role in the modulation of oxidative stress and cell growth, and it is associated with CD. ${ }^{83}$ While the minor allele $(G)$ increased risk of GI events by 2.5-fold in the colchicine group (HR: 2.51, p-value: $<0.001)^{79}$, the overall GI events were more likely to be reported by the carriers of one or 
two copies of the minor allele in the colchicine arm (47.1\%) than the placebo arm (18.9\%) (HR: 3.98 , p-value: $<0.001$ ). ${ }^{79}$

\section{Corticosteroids}

Corticosteroids (CORTs) are effective agents used to alleviate pain associated with acute gout flare, mainly when patients are intolerant of oral NSAIDs or colchicine. ${ }^{4}$ Due to the ability to administer these agents in various routes (oral, parenteral, or intra-articular), it allowed for expanded utilization in different clinical settings. CORTs act by blocking the eicosanoids production, leukotriene synthesis, and various leukocyte inflammatory processes by suppressing the phospholipase A2. ${ }^{4}$

Multiple genetic polymorphisms implicated in receptor binding (e.g., CRHR1, and NR3C1), Chaperone/Cochaperone protein (e.g., ST13, STIP1, and FKBP5), metabolizing enzymes (e.g., CYP3A4, CYP3A5, CYP3A7, and GSTT1), and transporters (e.g., ABCB1) ${ }^{84}$ have been linked to the response to CORTs and associated toxicities. CORTs are also substrates for P-gp, and hence polymorphisms in ABCB1 may result in different P-gp expressions, resulting in PK differences among CORTs or other transporter substrates. ${ }^{84,85}$

Today, there is no robust PGx data regarding the efficacy of CORTs in the management of gout flares; however, the pseudogene HLA complex group 22 (HCG22) was studied for PGx relevance in patients with retinal diseases and treated with intravitreal triamcinolone acetonide (injectable CORT). ${ }^{86}$ The SNPs rs3873352 C>G and rs2523864 C>T in HCG22, particularly the G and T alleles of each SNP, respectively, were linked to steroid-induced ocular hypertension $(\mathrm{OH})$ in triamcinolone users. ${ }^{86}$ All in all, CORT's molecular and metabolic pathways are daunting, and limited genomic factors with enough evidence for therapeutic applicability to gout have been established.

\section{IL-1 Inhibitors}

The members of the Interleukin-1 (IL-1) family are essential cytokines in the innate, and partly in adaptive immunity, and are found in high clusters in the articular tissue of human joints. ${ }^{87}$ The IL-1 alpha (i.e., IL-1F1) and IL-1 beta (i.e., IL-1F2) function as intracellular pro-inflammatory ligands for specific IL-1 complex receptors. ${ }^{87}$ The current and novel pharmacotherapies that target IL-1 include anakinra (recombinant IL-1 receptor antagonist "IL-1RN") and canakinumab (anti-IL-1 Beta), and rilonacept (IL-1 trap). Anakinra and canakinumab showed promising results in reducing gout flares. However, rilonacept was inferior to NSAID monotherapy over a 72-hour follow-up. ${ }^{87}$

A cluster of high-expression SNPs in the noncoding region of the interleukin-1 receptor antagonist $(I L-1 R N)$ gene were associated with poor response to anakinra. This SNP cluster included rs7580634 G>T, rs55709272 T>C, rs62158853 C>T, rs62158854 T>G, rs55663133 (deletion of the triplet AAT), and rs4251961 T>C. ${ }^{88}$ These six IL-1RN SNPs are inherited as a common haplotype in the studied sample. Furthermore, carriers of homozygous genotypes of one or more of the aforementioned SNPs had higher IL-1RN messenger RNA ( $I L-1 R N$ mRNA) and a 6-fold higher risk of nonresponse to anakinra. ${ }^{8}$ Although the associations between those SNPs and poor response to anakinra may seem intriguing, they were not studied in patients with gout.

\section{Conclusion}

Pharmacogenomics is gaining prominence as it could be used as a tool to improve the already existing low adherence to ULT in the context of gout management. The CPIC and FDA have identified several pharmacogenetic occurrences as having meaningful clinical significance in prescribing ULT and other medications used during acute gout flares. First, the risk of allopurinol-induced hypersensitivity (AHS), is significantly high in $H L A-$ $B * 58: 01$ carriers when taking allopurinol, whereas the risk of hemolysis and methemoglobinemia markedly increased when recombinant uricase is used in G6PD deficient patients. These occurrences are linked to significant morbidity and mortality; hence preemptive 
pharmacogenetic testing for $H L A-B^{*} 58: 01$ and G6PD are currently conditionally recommended for high-risk populations. Thirdly, CYP2C9*3/3 or *2/2 diplotypes are associated with prolonged exposure to ibuprofen and celecoxib, warranting dose reductions to prevent NSAID-induced GI bleeding risk. While preemptive genetic testing in gout management is underutilized, more pharmacogenomics research is needed to characterize its clinical utility compared to the standard of care. To optimize the response and safety of gout pharmacotherapies, healthcare professionals must be knowledgeable about relevant PGx occurrences to utilize preemptive PGx testing when applicable. (Table 1)

\section{Future perspective}

With the recent advancement in genetic research, personalized medicine could influence multiple facets of gout (e.g., stratifying disease risk, assessing disease progression, selecting and dosing of medications, and avoiding adverse drug reactions). The rapidly evolving technology in genetic sequencing has also facilitated significant landmark findings, leading to granular and novel gout-specific genetic discoveries. Though GWAS led to the identification of many genetic biomarkers of disease risk, specific knowledge gaps in the role of genetics in urate-lowering therapy persist. Collectively, well-characterized data that include the progression from hyperuricemia to gout, explicit drug information (e.g., dose-response outcomes and ADRs), and lifestyle factors will empower clinicians to personalize gout therapy and optimize gout prevention strategies. With most of the GWAS conducted on people of EUR descent, increasing the representation of diverse populations, particularly populations with a high prevalence of gout, is needed to move us beyond one genotype fits all. Additionally, the intricacies of the urate transportome and urate metabolism coupled with clinical factors implore a polygenic assessment approach to refine the prediction of response to gout pharmacotherapy and provide personalized dietary recommendations. Finally, a holistic gout treatment approach involving patientcentered outcomes, genetic information, lifestyle factors, and patient education could significantly improve gout management outcomes and increase adherence to ULT.

\section{References:}

1. Roden DM, McLeod HL, Relling M v., et al. Pharmacogenomics. Lancet (London, England). 2019;394(10197):521. doi:10.1016/S0140-6736(19)31276-0

2. Briesacher BA, Andrade SE, Fouayzi H, Chan KA. Comparison of drug adherence rates among patients with seven different medical conditions. Pharmacotherapy. 2008;28(4):437-443. doi:10.1592/PHCO.28.4.437

3. United Nations. Population I United Nations. Population. Accessed December 14, 2020. https://www.un.org/en/sections/issues-depth/population/

4. FitzGerald JD, Dalbeth N, Mikuls T, et al. 2020 American College of Rheumatology Guideline for the Management of Gout. Arthritis Care and Research. 2020;72(6):744-760. doi:10.1002/ACR.24180/ABSTRACT)

5. Butler F, Alghubayshi A, Roman Y. The epidemiology and genetics of hyperuricemia and gout across major racial groups: A literature review and population genetics secondary database analysis. Journal of Personalized Medicine. 2021;11(3). doi:10.3390/jpm11030231

6. Murdoch R, Barry MJ, Choi HK, et al. Original research: Gout, Hyperuricaemia and Crystal-Associated Disease Network (G-CAN) common language definition of gout. RMD Open. 2021;7(2):1623. doi:10.1136/RMDOPEN-2021001623

7. Kenny JES, Goldfarb DS. Update on the Pathophysiology and Management of Uric Acid Renal Stones. Current Rheumatology Reports 2010 12:2. 2010;12(2):125-129. doi:10.1007/S11926-010-0089-Y

8. Xu X, Huang J, Wu S, Ji Q, Guo X, Huang Y. The Association between the Serum Uric Acid Level and Hypertension in Middle-Aged and Elderly Adults. Cardiovascular Therapeutics. 2021;2021. doi:10.1155/2021/4626062 
9. Lanaspa MA, Andres-Hernando A, Kuwabara M. Uric acid and hypertension. Hypertension Research 2020 43:8. 2020;43(8):832-834. doi:10.1038/s41440-020-0481-6

10. Hu X, Rong S, Wang Q, et al. Association between plasma uric acid and insulin resistance in type 2 diabetes: A Mendelian randomization analysis. Diabetes Research and Clinical Practice. 2021;171:108542. doi:10.1016/J.DIABRES.2020.108542

11. Wright AF, Rudan I, Hastie ND, Campbell H. A “complexity” of urate transporters. Kidney international. 2010;78(5):446452. doi:10.1038/KI.2010.206

12. Yang Q, Köttgen A, Dehghan A, et al. Multiple genetic loci influence serum urate levels and their relationship with gout and cardiovascular disease risk factors. Circulation Cardiovascular genetics. 2010;3(6):523-530. doi:10.1161/CIRCGENETICS.109.934455

13. Enomoto A, Kimura H, Chairoungdua A, et al. Molecular identification of a renal urate anion exchanger that regulates blood urate levels. Nature. 2002;417(6887):447-452. doi:10.1038/NATURE742

14. Anzai N, Jutabha P, Amonpatumrat-Takahashi S, Sakurai H. Recent advances in renal urate transport: characterization of candidate transporters indicated by genome-wide association studies. Clinical and experimental nephrology. 2012;16(1):89-95. doi:10.1007/S10157-011-0532-Z

15. Woodward OM, Köttgen A, Coresh J, Boerwinkle E, Guggino WB, Köttgen M. Identification of a urate transporter, ABCG2, with a common functional polymorphism causing gout. Proceedings of the National Academy of Sciences of the United States of America. 2009;106(25):10338-10342. doi:10.1073/PNAS.0901249106

16. Caulfield MJ, Munroe PB, O'Neill D, et al. SLC2A9 is a high-capacity urate transporter in humans. PLoS medicine. 2008;5(10):1509-1523. doi:10.1371/JOURNAL.PMED.0050197

17. Vitart V, Rudan I, Hayward C, et al. SLC2A9 is a newly identified urate transporter influencing serum urate concentration, urate excretion and gout. Nature genetics. 2008;40(4):437-442. doi:10.1038/NG.106

18. FitzGerald JD, Dalbeth N, Mikuls T, et al. 2020 American College of Rheumatology Guideline for the Management of Gout. Arthritis and Rheumatology. 2020;72(6):879-895. doi:10.1002/art.41247

19. Hung SL, Chung WH, Liou LB, et al. HLA-B ${ }^{5} 501$ allele as a genetic marker for severe cutaneous adverse reactions caused by allopurinol. Proceedings of the National Academy of Sciences of the United States of America. 2005;102(11):41344139. doi:10.1073/pnas.0409500102

20. Avena-Woods C, Hilas O. Febuxostat (Uloric), A New Treatment Option for Gout. Pharmacy and Therapeutics. 2010;35(2):82. Accessed December 15, 2021./pmc/articles/PMC2827920/

21. Vora B, Brackman DJ, Zou L, et al. Oxypurinol pharmacokinetics and pharmacodynamics in healthy volunteers: Influence of BCRP Q141K polymorphism and patient characteristics. Clinical and translational science. 2021;14(4):14311443. doi:10.1111/CTS.12992

22. Stamp LK, Chapman PT. Allopurinol hypersensitivity: Pathogenesis and prevention. Best practice E research Clinical rheumatology. 2020;34(4). doi:10.1016/J.BERH.2020.101501

23. Stamp LK, Taylor WJ, Jones PB, et al. Starting dose is a risk factor for allopurinol hypersensitivity syndrome: a proposed safe starting dose of allopurinol. Arthritis and rheumatism. 2012;64(8):2529-2536. doi:10.1002/ART.34488

24. Hande KR, Noone RM, Stone WJ. Severe allopurinol toxicity. Description and guidelines for prevention in patients with renal insufficiency. The American journal of medicine. 1984;76(1):47-56. doi:10.1016/0002-9343(84)90743-5

25. Saito Y, Stamp LK, Caudle KE, et al. Clinical Pharmacogenetics Implementation Consortium (CPIC) guidelines for human leukocyte antigen B (HLA-B) genotype and allopurinol dosing: 2015 update. Clinical pharmacology and therapeutics. 2016;99(1):36-37. doi:10.1002/CPT.161 
26. Lu N, Rai SK, Terkeltaub R, Kim SC, Menendez ME, Choi HK. Racial disparities in the risk of Stevens-Johnson Syndrome and toxic epidermal necrolysis as urate-lowering drug adverse events in the United States. Seminars in Arthritis and Rheumatism. 2016;46(2):253-258. doi:10.1016/j.semarthrit.2016.03.014

27. Food \& Drug Administration. Table of Pharmacogenetic Associations I FDA. Accessed December 16, 2020. https://www.fda.gov/medical-devices/precision-medicine/table-pharmacogenetic-associations

28. Hershfield MS, Callaghan JT, Tassaneeyakul W, et al. Clinical Pharmacogenetics Implementation Consortium Guidelines for Human Leukocyte Antigen-B Genotype and Allopurinol Dosing. Clinical Pharmacology \& Therapeutics. 2013;93(2):153-158. doi:10.1038/clpt.2012.209

29. Carroll MB, Smith DM, Shaak TL. Genomic sequencing of uric acid metabolizing and clearing genes in relationship to xanthine oxidase inhibitor dose. Rheumatology international. 2017;37(3):445-453. doi:10.1007/S00296-016-3592-2

30. Wen CC, Yee SW, Liang X, et al. Genome-wide association study identifies ABCG2 (BCRP) as an allopurinol transporter and a determinant of drug response. Clinical pharmacology and therapeutics. 2015;97(5):518-525. doi:10.1002/CPT.89

31. Roman: THE IMPACT OF RS505802 FOR SLC22A12 ON OXIPURINOL... - Google Scholar. Accessed February 10, 2022. https://scholar.google.com/scholar?hl=en\&as_sdt=0,47\&cluster=16206836093874553445

32. Iwanaga T, Kobayashi D, Hirayama M, Maeda T, Tamai I. Involvement of uric acid transporter in increased renal clearance of the xanthine oxidase inhibitor oxypurinol induced by a uricosuric agent, benzbromarone. Drug metabolism and disposition: the biological fate of chemicals. 2005;33(12):1791-1795. doi:10.1124/DMD.105.006056

33. Anzai N, Ichida K, Jutabha $\mathrm{P}$, et al. Plasma urate level is directly regulated by a voltage-driven urate efflux transporter URATv1 (SLC2A9) in humans. The Journal of biological chemistry. 2008;283(40):26834-26838. doi:10.1074/JBC.C800156200

34. Roman YM, Culhane-Pera KA, Menk J, Straka RJ. Assessment of genetic polymorphisms associated with hyperuricemia or gout in the Hmong. Personalized medicine. 2016;13(5):429-440. doi:10.2217/PME-2016-0021

35. Alghubayshi A, Edelman A, Alrajeh K, Roman Y. Genetic assessment of hyperuricemia and gout in Asian, Native Hawaiian, and Pacific Islander subgroups of pregnant women: biospecimens repository cross-sectional study. BMC rheumatology. 2022;6(1). doi:10.1186/S41927-021-00239-7

36. Veenstra F, Wanten SAC, Verhoef LM, et al. Sex differences in response to allopurinol and benzbromarone in gout: a retrospective cohort study. Rheumatology advances in practice. 2021;5(1). doi:10.1093/RAP/RKAB002

37. Drug Approval Package: Uloric (Febuxostat) Tablets NDA 21856. Accessed December 15 , 2021. https://www.accessdata.fda.gov/drugsatfda_docs/nda/2009/021856s000toc.cfm

38. fda. HIGHLIGHTS OF PRESCRIBING INFORMATION. Accessed December 15, 2021. www.fda.gov/medwatch.

39. White WB, Saag KG, Becker MA, et al. Cardiovascular Safety of Febuxostat or Allopurinol in Patients with Gout. The New England journal of medicine. 2018;378(13). doi:10.1056/NEJMOA1710895

40. Zhang MA, Solomon DH, Desai RJ, et al. Assessment of Cardiovascular Risk in Older Patients With Gout Initiating Febuxostat Versus Allopurinol: Population-Based Cohort Study. Circulation. 2018;138(11):1116-1126. doi:10.1161/CIRCULATIONAHA.118.033992

41. Foody JA, Turpin RS, Tidwell BA, Lawrence D, Schulman KL. Major Cardiovascular Events in Patients with Gout and Associated Cardiovascular Disease or Heart Failure and Chronic Kidney Disease Initiating a Xanthine Oxidase Inhibitor. American Health E Drug Benefits. 2017;10(8):393. Accessed December 15, 2021. /pmc/articles/PMC5726059/

42. Barbarino JM, Haidar CE, Klein TE, Altman RB. PharmGKB summary: very important pharmacogene information for UGT1A1. Pharmacogenetics and genomics. 2014;24(3):177-183. doi:10.1097/FPC.0000000000000024

43. Bach MH, Simkin PA. Uricosuric drugs: the once and future therapy for hyperuricemia? Current opinion in rheumatology. 2014;26(2):169-175. doi:10.1097/BOR.0000000000000035 
44. Ichida K, Hosoyamada M, Hisatome I, et al. Clinical and molecular analysis of patients with renal hypouricemia in Japan-influence of URAT1 gene on urinary urate excretion. Journal of the American Society of Nephrology: JASN. 2004;15(1):164-173. doi:10.1097/01.ASN.0000105320.04395.D0

45. Hamada T, Ichida K, Hosoyamada M, et al. Uricosuric action of losartan via the inhibition of urate transporter 1 (URAT 1) in hypertensive patients. American journal of hypertension. 2008;21(10):1157-1162. doi:10.1038/AJH.2008.245

46. Pharmacogenomics Knowledge Base (PharmGKB). ABCB1 - Overview. Accessed December 16, 2020. https://www.pharmgkb.org/gene/PA267

47. Beringer PM, Kriengkauykiat J, Zhang X, et al. Lack of effect of P-glycoprotein inhibition on renal clearance of dicloxacillin in patients with cystic fibrosis. Pharmacotherapy. 2008;28(7):883-894. doi:10.1592/PHCO.28.7.883

48. Pharmacogenomics Knowledge Base (PharmGKB). Drug Label Information and Legend. Accessed December 16, 2020. https://www.pharmgkb.org/page/drugLabelLegend\#pgx-level

49. Chan TK, Todd D, Tso SC. Drug-induced haemolysis in glucose-6-phosphate dehydrogenase deficiency. British Medical Journal. 1976;2(6046):1227-1229. doi:10.1136/bmj.2.6046.1227

50. McDonagh EM, Thorn CF, Callaghan JT, Altman RB, Klein TE. PharmGKB summary: Uric acid-lowering drugs pathway, pharmacodynamics. Pharmacogenetics and Genomics. 2014;24(9):464-476. doi:10.1097/FPC.0000000000000058

51. Relling M v., Mcdonagh EM, Chang T, et al. Clinical Pharmacogenetics Implementation Consortium (CPIC) guidelines for rasburicase therapy in the context of G6PD deficiency genotype. Clinical pharmacology and therapeutics. 2014;96(2):169174. doi:10.1038/CLPT.2014.97

52. Pui CH. Rasburicase: A potent uricolytic agent. Expert Opinion on Pharmacotherapy. 2002;3(4):433-442. doi:10.1517/14656566.3.4.433

53. Pui CH, Relling M V., Lascombes F, et al. Urate oxidase in prevention and treatment of hyperuricemia associated with lymphoid malignancies. Leukemia. 1997;11(11):1813-1816. doi:10.1038/sj.leu.2400850

54. Pharmacogenomics Knowledge Base (PharmGKB). G6PD - Clinical Guideline Annotations. Accessed December 16, 2020. https://www.pharmgkb.org/gene/PA28469/guidelineAnnotation

55. Relling M V., Mcdonagh EM, Chang T, et al. Clinical Pharmacogenetics Implementation Consortium (CPIC) guidelines for rasburicase therapy in the context of G6PD deficiency genotype. Clinical Pharmacology and Therapeutics. 2014;96(2):169-174. doi:10.1038/clpt.2014.97

56. Beutler E, Gaetani G, Der Kaloustian V, Luzzatto L, Sodeinde O. Glucose-6-phosphate dehydrogenase deficiency. Bulletin of the World Health Organization. 1989;67(6):601-611. doi:10.32388/v63x86

57. Nkhoma ET, Poole C, Vannappagari V, Hall SA, Beutler E. The global prevalence of glucose-6-phosphate dehydrogenase deficiency: A systematic review and meta-analysis. Blood Cells, Molecules, and Diseases. 2009;42(3):267278. doi:10.1016/j.bcmd.2008.12.005

58. Tiitinen S, Nissilä M, Ruutsalo HM, Isomäki H. Effect of nonsteroidal anti-inflammatory drugs on the renal excretion of uric acid. Clinical rheumatology. 1983;2(3):233-236. doi:10.1007/BF02041396

59. PharmVar. Accessed January 15, 2022. https://www.pharmvar.org/gene/CYP2C9

60. Van Booven D, Marsh S, McLeod H, et al. Cytochrome P450 2C9-CYP2C9. Pharmacogenetics and Genomics. 2010;20(4):277281. doi:10.1097/FPC.0b013e3283349e84

61. Pharmacogenomics Knowledge Base (PharmGKB). Very Important Pharmacogene: CYP2C9. Accessed December 17, 2020. https://www.pharmgkb.org/vip/PA166169913

62. Theken KN, Lee CR, Gong L, et al. Clinical Pharmacogenetics Implementation Consortium Guideline (CPIC) for CYP2C9 and Nonsteroidal Anti-Inflammatory Drugs. CLINICAL PHARMACOLOGY E THERAPEUTICS I. doi:10.1002/cpt.1830 
63. Figueiras A, Estany-Gestal A, Aguirre C, et al. CYP2C9 variants as a risk modifier of NSAID-related gastrointestinal bleeding: a case-control study. Pharmacogenetics and Genomics. 2016;26(2):66. doi:10.1097/FPC.0000000000000186

64. Theken KN, Lee CR, Gong L, et al. Clinical Pharmacogenetics Implementation Consortium Guideline (CPIC) for CYP2C9 and Nonsteroidal Anti-Inflammatory Drugs. Clinical Pharmacology and Therapeutics. 2020;108(2):191-200. doi:10.1002/cpt.1830

65. Schlesinger N. Treatment of acute gout. Rheumatic diseases clinics of North America. 2014;40(2):329-341. doi:10.1016/J.RDC.2014.01.008

66. Slobodnick A, Shah B, Krasnokutsky S, Pillinger MH. Update on colchicine, 2017. Rheumatology (Oxford, England). 2018;57(suppl_1):i4-i11. doi:10.1093/RHEUMATOLOGY/KEX453

67. Slobodnick A, Shah B, Pillinger MH, Krasnokutsky S. Colchicine: old and new. The American journal of medicine. 2015;128(5):461-470. doi:10.1016/J.AMJMED.2014.12.010

68. Stewart S, Yang KCK, Atkins K, Dalbeth N, Robinson PC. Adverse events during oral colchicine use: a systematic review and meta-analysis of randomised controlled trials. Arthritis research \& therapy. 2020;22(1). doi:10.1186/S13075-020-21207

69. PharmVar. Accessed January 15, 2022. https://www.pharmvar.org/gene/CYP2D6

70. fda, cder. HIGHLIGHTS OF PRESCRIBING INFORMATION. Accessed January 15, 2022. www.fda.gov/medwatch.

71. Brown JT, Bishop JR, Sangkuhl K, et al. Clinical Pharmacogenetics Implementation Consortium Guideline for Cytochrome P450 (CYP)2D6 Genotype and Atomoxetine Therapy. Published online 2019:1. doi:10.1002/cpt.1409

72. Sohar E, Gafni J, Pras M, Heller H. Familial Mediterranean fever. A survey of 470 cases and review of the literature. The American Journal of Medicine. 1967;43(2):227-253. doi:10.1016/0002-9343(67)90167-2

73. Drenth JPH, van der Meer JWM. Hereditary Periodic Fever. New England Journal of Medicine. 2001;345(24):1748-1757. doi:10.1056/nejmra010200

74. Yalcintepe S, Ozdemir O, Silan C, et al. The CYP4502D6 ${ }^{*} 4$ and ${ }^{*} 6$ alleles are the molecular genetic markers for drug response: implications in colchicine non-responder FMF patients. European journal of drug metabolism and pharmacokinetics. 2016;41(3):281-286. doi:10.1007/S13318-015-0255-8

75. Babaoglu MO, Yasar U, Tufan A, et al. Association of the $3435 \mathrm{C}>\mathrm{T}$ polymorphism of the drug transporter gene ABCB1 with colchicine response in patients with familial Mediterranean fever. The FASEB Journal. 2007;21(5). doi:10.1096/FASEBJ.21.5.A414-D

76. Ozen F, Silan C, Uludag A, et al. Association between ABCB1 (MDR1) gene 3435 C $>$ T polymorphism and colchicine unresponsiveness of FMF patients. Renal failure. 2011;33(9):899-903. doi:10.3109/0886022X.2011.605980

77. Marzolini C, Paus E, Buclin T, Kim RB. Polymorphisms in human MDR1 (P-glycoprotein): recent advances and clinical relevance. Clinical pharmacology and therapeutics. 2004;75(1):13-33. doi:10.1016/J.CLPT.2003.09.012

78. Tardif JC, Kouz S, Waters DD, et al. Efficacy and Safety of Low-Dose Colchicine after Myocardial Infarction. The New England journal of medicine. 2019;381(26):2497-2505. doi:10.1056/NEJMOA1912388

79. Dubé MP, Legault MA, Lemaçon A, et al. Pharmacogenomics of the Efficacy and Safety of Colchicine in COLCOT. Circulation Genomic and precision medicine. 2021;14(2):223-229. doi:10.1161/CIRCGEN.120.003183

80. de Lange KM, Moutsianas L, Lee JC, et al. Genome-wide association study implicates immune activation of multiple integrin genes in inflammatory bowel disease. Nature genetics. 2017;49(2):256-261. doi:10.1038/NG.3760

81. SEPHS1 selenophosphate synthetase 1 [Homo sapiens (human)] - Gene - NCBI. Accessed February 4, 2022. https://www.ncbi.nlm.nih.gov/gene/22929

82. Gene symbol report I HUGO Gene Nomenclature Committee. Accessed February 4, 2022. https://www.genenames.org/data/gene-symbol-report/\#!/hgnc_id/HGNC:19685 
83. Gentschew L, Bishop KS, Han DY, et al. Selenium, selenoprotein genes and Crohn's disease in a case-control population from Auckland, New Zealand. Nutrients. 2012;4(9):1247-1259. doi:10.3390/NU4091247

84. Song QQ, Xie WY, Tang YJ, Zhang J, Liu J. Genetic variation in the glucocorticoid pathway involved in interindividual differences in the glucocorticoid treatment. Pharmacogenomics. 2017;18(3):293-316. doi:10.2217/PGS-2016-0151

85. corticosteroids. Accessed December 21, 2021. https://www.pharmgkb.org/chemical/PA10832/clinicalAnnotation

86. Jeong S, Patel N, Edlund CK, et al. Identification of a Novel Mucin Gene HCG22 Associated With Steroid-Induced Ocular Hypertension. Investigative ophthalmology \& visual science. 2015;56(4):2737-2748. doi:10.1167/IOVS.14-14803

87. Janssen CA, Oude Voshaar MAH, Vonkeman HE, et al. Anakinra for the treatment of acute gout flares: a randomized, double-blind, placebo-controlled, active-comparator, non-inferiority trial. Rheumatology (Oxford, England). 2019;58(8):1344-1352. doi:10.1093/RHEUMATOLOGY/KEY402

88. Pardeo M, Rossi MN, Pires Marafon D, et al. Early Treatment and IL1RN Single-Nucleotide Polymorphisms Affect Response to Anakinra in Systemic Juvenile Idiopathic Arthritis. Arthritis \& rheumatology (Hoboken, NJ). 2021;73(6):10531061. doi:10.1002/ART.41612 